\title{
Oestrogen receptor $\alpha$ is required for biochanin A-induced apolipoprotein A-1 mRNA expression in HepG2 cells
}

\author{
Ming Yan Chan ${ }^{1}$, Wai Man Gho ${ }^{1}$, Zhen-yu Chen ${ }^{1,2}$, Jun Wang ${ }^{1}$ and Lai K. Leung ${ }^{1,2} *$ \\ ${ }^{1}$ Department of Biochemistry, Faculty of Medicine \\ ${ }^{2}$ Food and Nutritional Sciences Programme, The Chinese University of Hong Kong, Room507C, MMW Bldg, \\ Shatin NT, Hong Kong
}

(Received 11 October 2006 - Revised 7 March 2007 - Accepted 7 March 2007)

\begin{abstract}
Epidemiological studies have indicated that soya consumption may produce a better plasma lipid profile. The effect may be attributed to the phyto-oestrogens in soya. The red clover (Trifolium pratense) isoflavone biochanin A has a chemical structure similar to those phyto-oestrogens found in soya beans, and is marketed as a nutraceutical for alleviating postmenopausal symptoms. In the present study we investigated the effect of biochanin A on the mRNA expression of ApoA-1 in the hepatic cell line HepG2. Real-time PCR revealed that biochanin A increased ApoA-1 mRNA abundance in cells expressing oestrogen receptor (ER) $\alpha$. Without ER $\alpha$ transfection, biochanin A had no effect on mRNA abundance. In order to study the transcriptional control, a fragment of the $5^{\prime}$-flanking region of the ApoA-1 gene was amplified and inserted in a firefly luciferase reporter plasmid. The reporter assay indicated that the transactivation of the ApoA-1 promoter was induced by biochanin A in HepG2 cells transfected with the ER $\alpha$ expression plasmid. This induction was reduced by the anti-oestrogen ICI 182,780, whereas the inhibitors of protein kinase (PK) C, PKA, or mitogen-activated kinase (ERK) had no suppressive effect. The present study illustrated that biochanin A might up regulate hepatic apoA-1 mRNA expression through an ER-dependent pathway.
\end{abstract}

Biochanin A: Oestrogen receptors: Apolipoprotein A-1: Liver cancer cells

CVD comprises the major cause of death in Western countries. Recent projections suggest that CVD will be the leading cause of death in both developed and developing regions of the world by the year $2020^{1}$. Epidemiological studies have associated the consumption of isoflavonoids with a lower incidence of $\mathrm{CVD}^{2}$. In normal postmenopausal women, consuming whole soya foods with $60 \mathrm{mg}$ isoflavones per d may help to alleviate several key clinical risk factors for $\mathrm{CVD}^{3}$.

HDL is synthesised in hepatic and intestinal cells and secreted as small particles containing phospholipids, nonesterified cholesterol, ApoA-1 and ApoE. Cholesterol synthesised or deposited in peripheral tissues is returned to the liver in a process referred to as 'reverse cholesterol transport'. ApoA-1 activates lecithin-cholesterol acyltransferase and facilitates the removal of cholesterol from the tissues (for a review, see Fielding \& Fielding ${ }^{4}$ ).

Oestrogen receptor (ER) $\alpha$ is a member of nuclear hormone receptors for binding a wide range of hydrophobic molecules, such as steroid hormone and phyto-oestrogens. ER $\alpha$ is found in various tissues, including the liver, bone, heart and central nervous system ${ }^{5}$. Oestrogen binds to the C-terminal domain of $\mathrm{ER} \alpha$ in the cytoplasm and releases the heat-shock proteins. The activated ER $\alpha$ is translocated into the nucleus and seeks out genes with specific response elements for binding.
The gene transcription machinery is then activated and the encoded mRNA is expressed.

Isoflavones are one group of the major phyto-oestrogens that have been the focus of many studies regarding their health benefits. Isoflavones share some common structure with the hormone oestrogen. Despite the similarity, the relative binding affinity of isoflavones for $\mathrm{ER} \alpha$ is only $0.05-1 \%$ of the binding affinity of $17 \beta$-oestradiol $\left(E_{2}\right)^{6}$. In contrast, their binding affinity for ER $\beta$ is approximately seven-fold greater than that of oestrogen. It is suggested that isoflavones may act as selective ER modulators ${ }^{7}$. In addition, the plasma isoflavone concentration can be several thousand times greater than that of $\mathrm{E}_{2}{ }^{8}$. They may compete for $\mathrm{ER}$ and display anti-oestrogenic effects, especially when endogenous oestrogen level is low. Biochanin A $(5,7$ dihydroxy-4'methoxyisoflavone) can be isolated from red clover (Trifolium pratense), and is a nutraceutical for relieving postmenopausal symptoms. The oestrogenic activity of biochanin A is several orders of magnitude lower when compared with other structurally related isoflavones, such as genistein and daidzein ${ }^{9}$.

Previous studies ${ }^{10-12}$ have shown that HepG2 cells can be a viable model for apolipoprotein research except that these cells do not express $\mathrm{ER} \alpha^{13}$. By using this cell model, the

\footnotetext{
Abbreviations: E2, oestradiol; ER, oestrogen receptor; HNF, hepatocyte nuclear factor; MTT, 3-(4, 5-dimethylthiazol-2-yl)-2, 5-diphenyl tetra-zolium bromide; PKA, protein kinase A; PKC, protein kinase C.

* Corresponding author: Dr Lai K. Leung, fax +852 26037732, email laikleung@yahoo.com
} 
present study was designed to investigate the regulatory mechanism of biochanin A on ApoA-1.

\section{Materials and methods}

Chemicals

Biochanin A was purchased from Sigma Chemicals (St Louis, MO, USA). PD98059, bisindolylmaleimide I and myristoylated PK inhibitor (PKI) 14-22 amide were obtained from EMD Biosciences Inc. (La Jolla, CA, USA). All other chemicals, if not stated, were acquired from Sigma Chemicals.

\section{Cell culture}

HepG2 cells (American Tissue Culture Collection, Rockville, MD, USA) were routinely cultured in RPMI-1640 media (Sigma Chemicals), supplemented with $10 \%$ fetal bovine serum (Invitrogen Life Technology, Rockville, MD, USA) and antibiotics penicillin $(50 \mathrm{U} / \mathrm{ml})$ and streptomycin $(50 \mu \mathrm{g} /$ $\mathrm{ml}$ ), and incubated at $37^{\circ} \mathrm{C}$ and $5 \%$ carbon dioxide. At $3 \mathrm{~d}$ before the experiment, the cultures were switched to RPMI1640 phenol red-free media (Sigma Chemicals) and $5 \%$ charcoal-dextran-treated fetal bovine serum (Hyclone, UT, USA). Sub-confluent cell cultures were treated with isoflavone with dimethyl sulfoxide as the carrier solvent. The final concentration of the solvent was $0 \cdot 1 \%(\mathrm{v} / \mathrm{v})$, and the control cultures received dimethyl sulfoxide only.

\section{Luciferase reporter gene assay}

Construction of ApoA-1-driven luciferase reporter plasmids. Fragments with $2361 \mathrm{bp}$ (ApoA-1: -2068 to +293$), 761 \mathrm{bp}$ (468del: -468 to +293$)$, 536 bp (243del: -243 to +293$)$ and 333 bp (40del: -40 to +293 ) from human ApoA-1 5'-flanking region were amplified from genomic DNA isolated from HepG2 cells. Primers were designed with the incorporation of Mlu I and Bgl II restriction sites. The forward primers for the respective constructs were: pTA-ApoA-1-luc, 5'-CCG ACG CGT GGT ACC TTT CTA ACA GTT TTG-3'; pTA-468delluc, 5'-TTC GAC GCG TAC TAA AGA AGA GCA CTG G3'; pTA-243del-luc, 5'-TTT AAC GCG TCT GCA AGC CTG CAG CAC T-3'; pTA-40del-luc, 5'-AAG GAC GCG TGG CTG CAG ACA TAA ATA G-3'. They all shared the common reverse primer, 5'-ACA AGA TCT TTA GGG GAC ACC TAC CCG TCA-3'. The amplified product was then digested and subcloned into a firefly luciferase reporter vector pTA-Luc (BD Biosciences Clontech, Palo Alto, CA, USA), and the sequence accuracy was verified.

Dual luciferase assays. HepG2 cells were seeded at $10^{5}$ cells/well in twenty-four-well plates. After $24 \mathrm{~h}$, the cells were transiently transfected with the reporter and ER expression plasmid at $0.4 \mu \mathrm{g}$ each and $0.1 \mu \mathrm{g}$ of renilla luciferase control vector pRL (Promega, Madison, WI, USA) in LipofectAmine (Invitrogen Life Technologies). ER $\alpha$ and ER $\beta$ expression plasmids were generous gifts from Dr Donald Macdonald (Duke University, Durham, NC, USA). After $16 \mathrm{~h}$, the medium was removed and the cells were treated with isoflavone for $24 \mathrm{~h}$. The amounts of these two luciferases were determined using the Dual-Luciferase Assay Kit (Promega). The luciferase bioluminescence was quantified by using a FLUOstar Galaxy plate reader. The transactivation activities of the $A p o A-1$ promoter represented by firefly luciferase light units were then normalised with that of renilla luciferase.

Quantitative real-time reverse transcriptase-polymerase chain reaction assay

HepG2 cells were seeded in a six-well plate for $1 \mathrm{~d}$ and transfected with $E R \alpha$ expression plasmid or the empty vector pcDNA3 1 . The medium was removed and cells were cultured with biochanin A. After $24 \mathrm{~h}$ of treatment, total RNA was extracted from the cells using TRIzol reagent (Invitrogen, Carlsbad, CA, USA). The concentration and purity of RNA were determined by absorbance at $260 / 280 \mathrm{~nm}$. First, DNA strands were synthesised from $3 \mu \mathrm{g}$ total RNA using oligodT primers and Moloney murine leukaemia virus RT (M-MLV RT; USB Corporation, Cleveland, OH, USA). Target fragments were quantified by real-time PCR, and a DNA Engine Opticon ${ }^{\mathrm{TM}} 2$ (MJ Research, Watertown, MA, USA) was employed for this assay. Taqman ${ }^{\circledR}-$ VIC $^{\circledR}$ MGB probes and primers for apoA-1 and GAPDH (Assay-onDemand $^{\mathrm{TM}}$ ) and real-time PCR Taqman Universal PCR Master Mix were all obtained from Applied Biosystems (Foster City, CA, USA). PCR reactions were set up as described in the protocol, which was validated by the company. Signals obtained for GAPDH (used as a reference housekeeping gene) were used to normalise the amount of total RNA amplified in each reaction. Relative gene expression data were analysed using the $2^{-\Delta \Delta C} \mathrm{~T}$ method ${ }^{14}$.

\section{Western analysis}

Cells were washed once by PBS ( $\mathrm{pH} 7.4)$ and harvested into a $1.5 \mathrm{ml}$ microtube with $0.5 \mathrm{ml}$ lysis buffer (PBS, $1 \%$ Nonidet $^{\circledR}$ P-40 (NP-40), 0.5\% sodium deoxycholate and 0.1\% SDS). The lysis buffer contained protease inhibitors (PMSF $(40 \mathrm{mg}$ / 1), aprotinin $(0.5 \mathrm{mg} / \mathrm{l})$, leupeptin $(0.5 \mathrm{mg} / \mathrm{l}), 1.1 \mathrm{mM}$-EDTA and pepstatin $(0.7 \mathrm{mg} / \mathrm{l}))$. The harvested cells were then lysed with a cell disruptor (Branson Ultrasonics Corp., Danbury, CT, USA) on ice for $30 \mathrm{~s}$. The protein concentration of cell lysate was determined by Dc protein assay (BioRad, Richmond, CA, USA). Lysate protein $(50 \mu \mathrm{g})$ was separated on $10 \%$ SDSPAGE and transferred onto an Immobilon PVDF membrane (Millipore, Bedford, MA, USA). For the Western analysis performed on culture medium, the volume loaded was normalised with the 3-(4, 5-dimethylthiazol-2-yl)-2, 5-diphenyl tetrazolium bromide (MTT) absorbance reading. Anti-ApoA-1 (Santa Cruz Biotechnology, Santa Cruz, CA, USA), anti-actin primary (Sigma Chemicals) and secondary antibodies conjugated with horseradish peroxidase (Santa Cruz Biotechnology) were used for protein detection. An ECL Detection Kit (Amersham, Arlington Heights, IL, USA) provided the chemiluminescence substrate for horseradish peroxidase, and the targeted protein was visualised by autoradiography.

\section{Measurement of cell viability}

Cell number was assessed by MTT staining as described by $\operatorname{Mosmann}^{15}$. Briefly, HepG2 cells were seeded in a ninety-six-well plate and were transfected with $\mathrm{ER} \alpha$ and 
treated as described earlier. At the end of the treatment, $1500 \mu \mathrm{l} \mathrm{MTT}(1 \mathrm{mg} / \mathrm{ml})$ were added to the cells and incubated at $37^{\circ} \mathrm{C}$ for $4 \mathrm{~h}$. Cell viability was assessed with respect to the absorbance at $544 \mathrm{~nm}$.

\section{Statistical methods}

A Prism ${ }^{\circledR} 3.0$ software package (GraphPad Software, Inc., CA, USA) was utilised for statistical analysis. Results of the present study were compared by ANOVA and Bonferroni's method for multiple comparisons. Significance level was set at $P<0 \cdot 05$.

\section{Results}

Effect of biochanin A on apolipoprotein A-1 mRNA expression

Cultures transfected with $\mathrm{ER} \alpha$ had a dose-dependent increase in ApoA-1 mRNA abundance upon biochanin A treatment, whereas the apoA-1 expression in cells transfected with the empty vector was not affected by the same treatment (Fig. 1). Biochanin A at concentrations of 1 and $10 \mu \mathrm{M}$ could induce 3- to 5-fold increases in mRNA abundance, whereas $E_{2}$ at $1 \mathrm{nM}$ elicited an increase of about 7-fold. No induction was observed in empty vector-transfected cultures.

Response of ApoA-1 promoter to biochanin A in HepG2 cells expressing oestrogen receptor $\alpha$

Following the real-time PCR experiment, we carried out reporter gene assays to verify the expression regulation. Significant elevations in the $A p o A-1$ promoter-driven luciferase activity were demonstrated in ER-transfected cells treated with biochanin A at $0.5 \mu \mathrm{M}$ or above (Fig. 2), and we observed increased activities ranging from 300 to $600 \%$. $\mathrm{E}_{2}$ at the concentration of $1 \mathrm{~nm}$ also induced a 3 -fold increase in the normalised luciferase activities compared with the control.

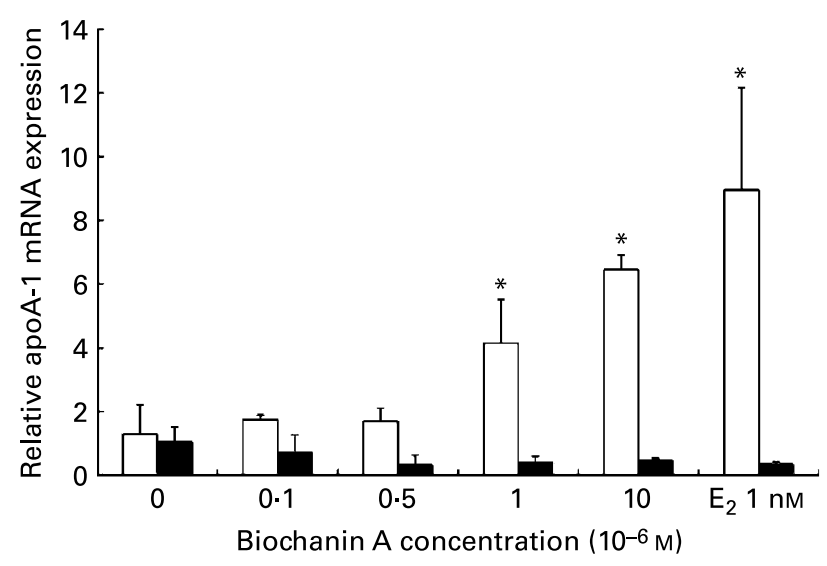

Fig. 1. Effect of biochanin $A$ on ApoA-1 mRNA expression in the presence $(\square)$ and absence $(\square)$ of oestrogen receptor (ER) $\alpha$ in HepG2 cells. HepG2 cells were transfected with ER $\alpha$ expression plasmid or pcDNA3.1 vector. After $1 \mathrm{~d}$, cells were treated with biochanin $A$ or $1 \mathrm{~nm}$-oestradiol $\left(E_{2}\right)$. Total RNA was isolated and the ApoA-1 expression was measured. Values are means, with standard deviations represented by vertical bars. * Mean expression was significantly increased when compared with that of the control $(P<0.05)$.

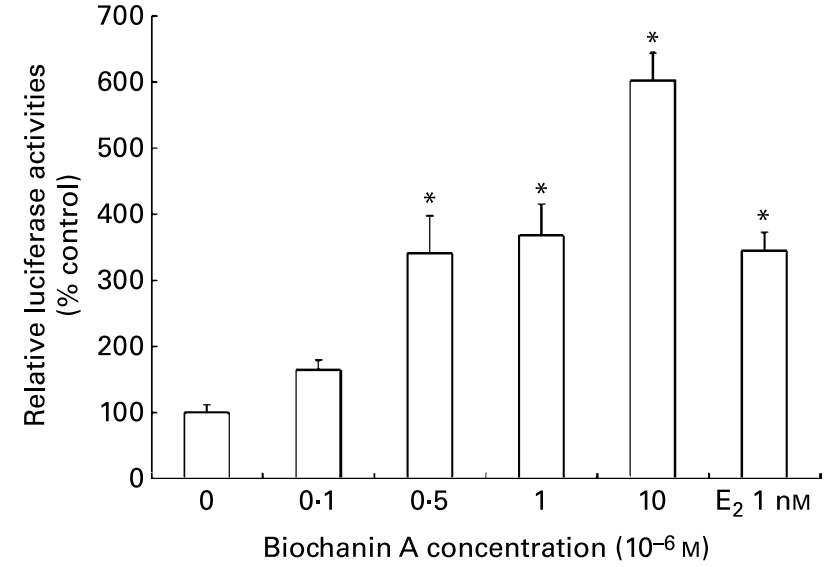

Fig. 2. Biochanin $A$ increased $A p o A-1$ promoter transactivation in HepG2 cells expressing oestrogen receptor (ER) $\alpha$. HepG2 cells were plated and transfected with pTA-ApoA-1-luc, ER $\alpha$ expression plasmid and the control plasmid $p R L$. Cells were treated with biochanin $A$ or oestradiol $\left(E_{2}\right)$ for $24 \mathrm{~h}$. Cell extracts were analysed for luciferase activity. Values are means, with standard deviations represented by vertical bars. * Mean expression was significantly increased when compared with that of the control $(P<0.05)$.

\section{Effect of isoflavone on apolipoprotein A-1 protein}

ApoA-1 expression at the protein level was also assessed in cell lysates and culture medium. However, the ApoA-1 protein in cell lysates or culture medium was not increased by biochanin A treatment (Fig. 3). It was possible that the translational machinery was unable to cope with the increased messages in this cell system.
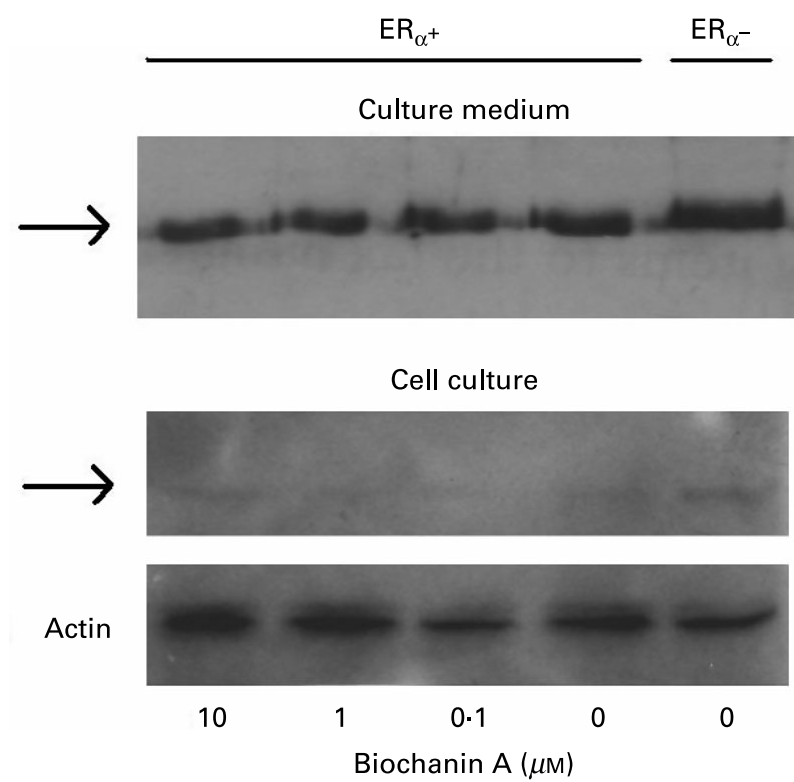

Fig. 3. Western analysis of ApoA-1 protein in cultures and culture medium under biochanin A treatment. HepG2 cells were transfected with oestrogen receptor $(E R) \alpha$ and treated with biochanin $A$. Western analysis was performed on cell lysates and culture medium. The autoradiographs are shown; the culture medium was normalised with the respective viable cell number (3-(4, 5-dimethylthiazol-2-yl)-2, 5-diphenyl tetra-zolium bromide (MTT) absorbance). The blots are representatives of two independent experiments with similar results. 
Effect of protein kinase inhibitors and anti-oestrogen on ApoA-1 promoter-driven luciferase activities in cultures expressing oestrogen receptor $\alpha$

Previous studies ${ }^{16,17}$ have indicated the involvement of protein kinase A (PKA), protein kinase C (PKC) or mitogen-activated protein kinase in ApoA-1 regulation. Protein kinase inhibitor (PKI) 14-22 amide, bisindolylmaleimide I and PD98059 are specific inhibitors for PKA, PKC and mitogen-activated protein kinase kinase, respectively. The inhibitor concentrations have been validated in HepG2 cells ${ }^{16}$. Compared with the control the administration of these inhibitors did not substantially decrease the ApoA-1 promoter-driven gene transactivation (Fig. 4(A)). PD98059 could induce rather than suppress the promoter activity. When the pure anti-oestrogen ICI 182,730 was administered, luciferase activity was significantly $(P<0.05)$ reduced as demonstrated in Fig. 4(B). These data illustrated that $\mathrm{ER} \alpha$ was involved in biochanin A-induced ApoA-1 transcriptional activation.
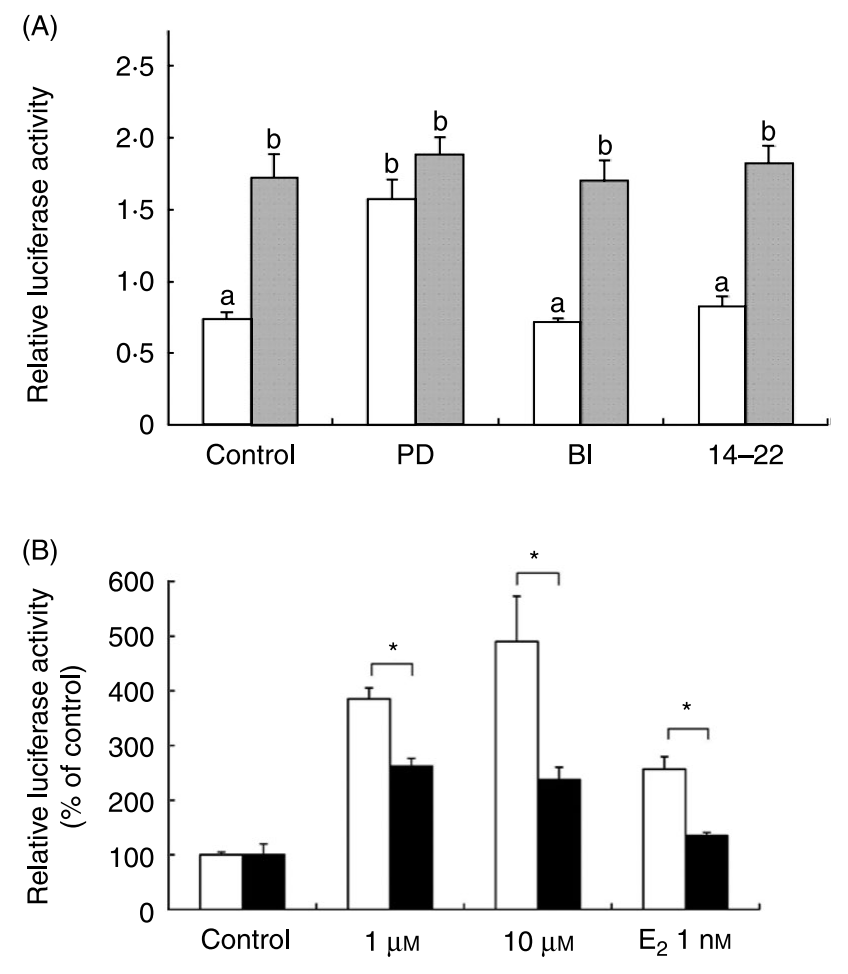

Biochanin A concentration

Fig. 4. Effect of inhibitors of protein kinase $C$, protein kinase $A$, mitogen-activated protein kinase and $\mathrm{ICl} 182,780$ on biochanin A-induced ApoA-1 promoter transactivation. HepG2 cells were transiently transfected with pTAApoA-1-luc plasmid and oestrogen receptor $\alpha$ expression plasmid for $24 \mathrm{~h}$. (A) Following transfection, cells were pretreated for $1 \mathrm{~h}$ with $10 \mu \mathrm{M}-\mathrm{PD}$ 98059 (PD), $10 \mu \mathrm{M}$-myristoylated protein kinase inhibitor (PKI) 14-22 amide (14-22) and $1 \mu \mathrm{M}$-bisindoylmaleimde I (BI). Biochanin A $(1 \mu \mathrm{M} ; \square)$ or dimethyl sulfoxide $(\square)$ was then administered. Values are means, with standard deviations represented by vertical bars. ${ }^{\mathrm{a}, \mathrm{b}}$ Mean values with unlike letters were significantly different $(P<0.05)$. (B) Biochanin A or $1 \mathrm{~nm}$-oestradiol $\left(E_{2}\right)$ was administered with $(\square)$ or without $(\square)$ pretreatment of $I C I$ 182,780 for $1 \mathrm{~h}$. Cell extracts were collected after $24 \mathrm{~h}$ for the determination of luciferase activity. Values are means, with standard deviations represented by vertical bars. * Mean activity was significantly decreased when compared with the biochanin $\mathrm{A}$ - or $\mathrm{E}_{2}$-treated cultures $(P<0.05)$.
Determination of ApoA-1 promoter-driven luciferase activities in oestrogen receptor $\alpha$-transfected HepG 2 cells

HepG2 cells were transfected with various ApoA-1 reporter constructs and $\mathrm{ER} \alpha$ expression plasmid. Luciferase activity was subsequently measured to reveal the transcriptional control of apoA-1 expression. All ApoA-1 constructs displayed increasing trends when treated with increased concentrations of biochanin A (Fig. 5(A)). The greatest response was observed in the pTA-ApoA-1-luc construct. However, the increases of the luciferase signals were similar among different biochanin A concentrations within the same construct in terms of the percentage induced. This indicated that no critical promoter segment was induced by biochanin A (Fig. 5(B)).

Investigation of ApoA-1 promoter-driven luciferase activities in HepG2 cells expressing oestrogen receptor $\beta$

Since ER $\beta$ might also activate oestrogen response element, we carried out another reporter gene assay to determine whether

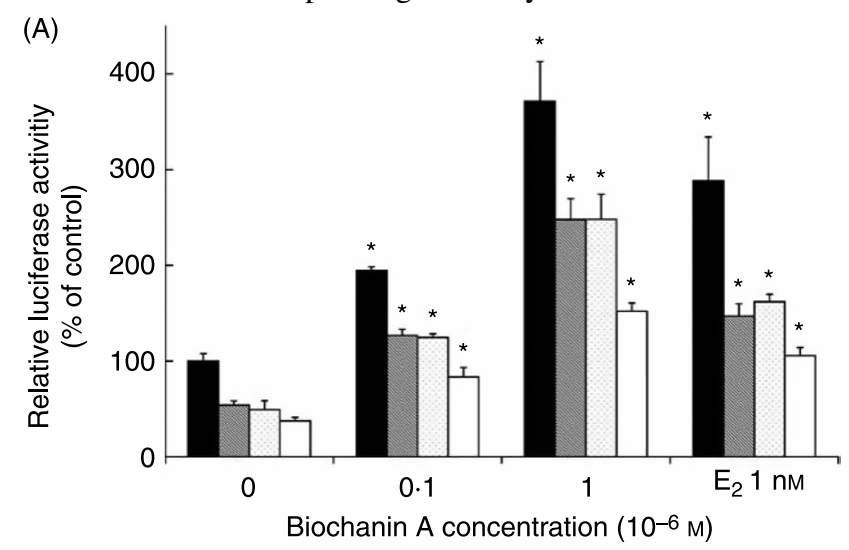

(B)

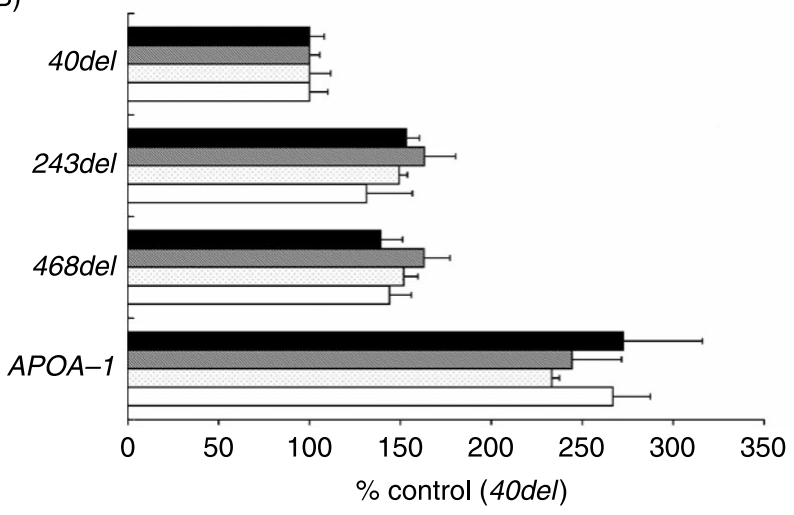

Fig. 5. Biochanin A-induced ApoA-1 promoter activity profile. HepG2 cells were seeded in twenty-four-well culture plates and transfected with the serial truncation plasmid, oestrogen receptor $\alpha$ expression plasmid, and renilla luciferase plasmid. After $24 \mathrm{~h}$ of transfection, the cultures were treated with biochanin A for each construct. The cells were lysed and assayed for firefly and renilla luciferase activities. (A) One set of two experiments performed

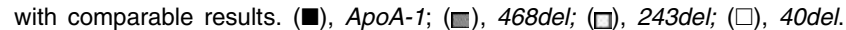
Values are means, with standard deviations represented by vertical bars. * Mean activity was significantly increased when compared with the control cultures $(P<0.05)$. (B) Replot of the values for each of the truncated ApoA-1 promoter normalised with its own construct without biochanin $A$ treatment.

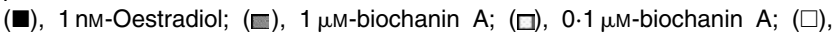
$0 \mu \mathrm{M}$-biochanin $A$. Values are are means $(n 3)$, with standard deviations represented by vertical bars. 
the expression was also regulated by this ER isoform. Biocha$\operatorname{nin} \mathrm{A}$ at $10 \mu \mathrm{M}$ showed a marginal elevation in the normalised luciferase activities (Fig. 6), and the increased activities were not deviated from the empty vector. The $A p o A-1$ promoterdriven luciferase activity was not significantly increased by biochanin $\mathrm{A}$ in cells expressing ER $\beta$.

\section{Discussion}

In the present study, we found that biochanin A up regulated apoA-1 mRNA expression in HepG2 cells expressing $\mathrm{ER} \alpha$, not ER $\beta$. ApoA-1 promoter-driven reporter gene assays supported that the up regulation was introduced by increased transcriptional activities. The induction pathway appeared to be independent of mitogen-activated kinase (ERK), PKA and PKC. Luciferase assays using the truncation reporter plasmids also did not reveal any critical elements lying between -40 and -2068 in the $5^{\prime}$-flanking region of the ApoA-1 promoter.

$17 \beta-\mathrm{E}_{2}$ and genistein have been shown to increase the promoter activities of $A p o A-1$ in HepG 2 cells ${ }^{18,19}$. Similarly, the present study demonstrated that biochanin A activated both the ApoA-1 mRNA expression and ApoA-1 promoter activity. The phyto-oestrogen biochanin A appeared to activate ER $\alpha$ for the induction of mRNA expression of ApoA-1, and the condition has not been established in the above-mentioned studies. It has been shown that the mitogen-activated kinase (ERK) activation pathway is increased in the up regulation of ApoA-1 gene expression by genistein and $\mathrm{E}_{2}$ in wild-type HepG2 cells ${ }^{16}$. Conversely, Beers et al. ${ }^{17}$ have shown that overexpressing ERK1/2 suppresses rather than encourages the transcriptional activities. The present study indicated that inhibition of several signalling pathways including the mitogen-activated protein kinase, PKC and PKA pathways did not abolish the augmented ApoA- 1 transcription. Mitogen-activated protein kinase inhibitor by itself might even increase the transactivity, which could be contradictory to the above-mentioned observations. This suggested that the up regulation of ApoA-1 transcriptional activity in the presence of $\mathrm{ER} \alpha$ was probably not going through these signalling pathways.

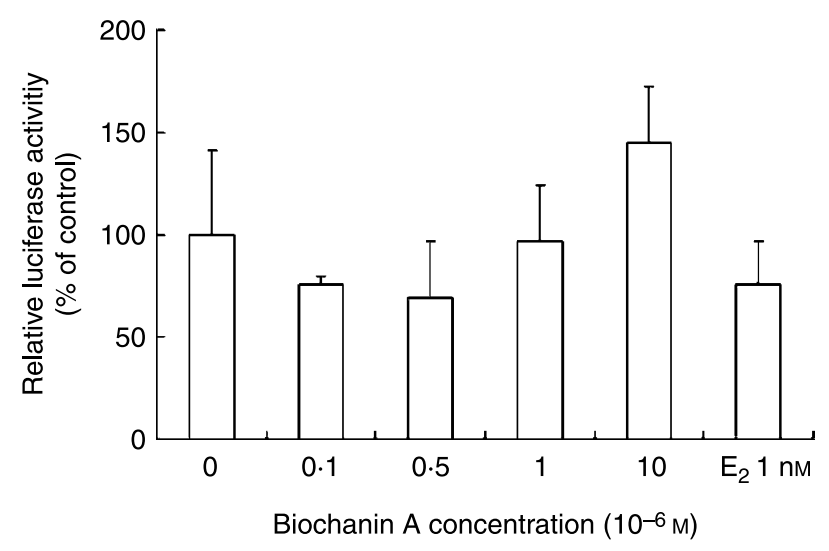

Fig. 6. Effect of biochanin A on $A p o A-1$ promoter transactivation in HepG2 cells expressing oestrogen receptor (ER) $\beta$. HepG2 cells were plated and transfected with pTA-ApoA-1-luc, ER $\beta$ expression plasmid and the control plasmid $p R L$. Cells were treated with biochanin $A$ or oestradiol $\left(E_{2}\right)$ for $24 \mathrm{~h}$. Cell extracts were analysed for luciferase activity. Values are means, with standard deviation represented by vertical bars.
The first $256 \mathrm{bp}$ upstream in the $5^{\prime}$-flanking region is critical in $A p o A-1$ regulation in HepG2 cells. Unlike the intestinal Caco-2 cells which require the segment -192 to -2052 for transcription, the segment between -41 and -256 is sufficient and specific for maximal ApoA-1 transcription in HepG2 cells $^{20}$. Previous studies have shown that the increase in ApoA-1 gene expression by oestrogen and genistein is mediated through the -256 to -41 region of the ApoA-1 promoter ${ }^{18,19}$. This region contains binding sites for three transcription factors, which are hepatocyte nuclear factors (HNF)-3 $\beta$, HNF-4 and early growth response factor Egr-1. Sites at -214 to -192 and -169 to -146 have been shown to contain response elements for HNF-4 ${ }^{21}$ and HNF$3 \beta^{22}$, respectively. Binding sites for Egr-1 have also been located at -221 to -231 and -189 to $-181^{23}$. Our findings did not support the notion that oestrogen increased ApoA1 mRNA expression in HepG2 cells without ER $\alpha$. With respect to our truncation reporter experiments performed in HepG2 cells expressing ER $\alpha$, biochanin A treatment did not initiate a higher luciferase response than the control in cells transfected with reporter plasmids constructed by deleting sequences from -2068 to -40 . Hence, the cis-acting DNA binding sites activated by biochanin A was apparently not present in this segment. Because an increase in promoter activity was observed in the reporter assays, biochanin A might activate an enhancer element or deactivate a repressor element in a region further upstream or downstream of the gene. A $48 \mathrm{bp}$ enhancer element locating at the far distal region of ApoA-1 (+8446/+8399) has been identified ${ }^{24}$, which could be a possible activation pathway in the present study.

Oestrogen replacement therapy has long been used for controlling postmenopausal symptoms, including lowering blood cholesterol. Lamon-Fava et al. ${ }^{18}$ have demonstrated that $\mathrm{E}_{2}$ increases promoter activities of ApoA-1 in HepG2 cells. An ER-independent pathway has also been described by Zhang et $a l^{25}$ for equine oestrogen in the up regulation of $A p o A-1$ promoter activity. In contrast, oestrogen may repress ApoA1 expression. Harnish et al. ${ }^{13}$ observed that $100 \mathrm{~nm}-\mathrm{E}_{2}$ represses ApoA-1 promoter activity in HepG2 cells stably expressing $\mathrm{ER} \alpha$. These contradictory observations can be explained by differences in the oestrogen concentration, timing, or the model nature.

\section{Acknowledgements}

The present study was supported by the Chinese University of Hong Kong Direct Grant for Research (code no. 2041184).

\section{References}

1. Murray JL \& Lopez AD (1996) The Global Burden of Disease: A Comprehensive Assessment of Global Mortality and Disability from Diseases, Injuries and Risk Factors in 1990 and Projected to 2020. Geneva: World Health Organization.

2. Adlercreutz H, Markkanen H \& Watanabe S (1998) Plasma concentrations of phyto-oestrogens in Japanese men. Lancet 342, 1209-1210.

3. Scheiber MD, Liu JH, Subbiah MTR, Rebar RW \& Setchell KDR (2001) Dietary inclusion of whole soy foods results in significant reductions in clinical risk factors for osteoporosis and 
cardiovascular disease in normal postmenopausal women. Menopause 8, 384-392.

4. Fielding CJ \& Fielding PE (1995) Molecular physiology of reverse cholesterol transport. J Lipid Res 36, 211-228.

5. Gustafsson JA (1999) Estrogen receptor $\beta$ - a new dimension in estrogen mechanism of action. $J$ Endocrinol 163, 379-383.

6. Shutt DA \& Cox RI (1972) Steroid and phyto-estrogen binding to sheep uterine receptors in vitro. J Endocrinol 52, 291-310.

7. Kuiper GGJM, Lemmen JG, Carlsson B, Corton JC, Safe SH, van der Saag PT, van der Burg B \& Gustafsson JA (1998) Interaction of estrogenic chemicals and phytoestrogens with estrogen receptor $\beta$. Endocrinology. 139, 4252-4263.

8. Adlercreutz H (2003) Phytoestrogens and breast cancer. J Steroid Biochem Mol Biol 83, 113.

9. Jefferson WN, Padilla-Banks E, Clark G \& Newbold RR (2002) Assessing estrogenic activity of phytochemicals using transcriptional activation and immature mouse uterotrophic responses. J Chromatogr B Analyt Technol. Biomed Life Sci 777, 179.

10. Javitt NB (1990) HepG2 cells as a resource for metabolic studies: lipoprotein, cholesterol and bile acids. FASEB J 4, 161-168.

11. Rash JM, Rothblat GH \& Sparks CE (1981) Lipoprotein apolipoprotein synthesis by human hepatoma cells in culture. Biochim Biophys Acta 666, 294-298.

12. Zannis VI, Breslow JL, SanGiacomo TR, Aden DP \& Knowles BB (1981) Characterization of the major apolipoproteins secreted by two human hepatoma cell lines. Biochemistry 20, 7089-7096.

13. Harnish DC, Evans MJ, Scicchitano MS, Bhat RA \& Karathanasis SK (1998) Estrogen regulation of the apolipoprotein AI gene promoter through transcription cofactor sharing. J Biol Chem 273, 9270-9278.

14. Livak KJ \& Schmittgen TD (2001) Analysis of relative gene expression data using real-time quantitative PCR and the 2(-Delta Delta C(T)) method. Methods 25, 402-408.

15. Mosmann T (1983) Rapid colorimetric assay for cellular growth and survival: application to proliferation and cytotoxicity assays. J Immunol Methods 65, 55-63.
16. Lamon-Fava S \& Micherone D (2004) Regulation of apoA-I gene expression mechanism of action of estrogen and genistein. J Lipid Res 45, 106-112.

17. Beers A, Haas MJ, Wong NCW \& Mooradian AD (2006) Inhibition of apolipoprotein AI gene expression by tumor necrosis factor $\alpha$ : roles for MEK/ERK and JNK signaling. Biochemistry 45, 2408-2413.

18. Lamon-Fava S, Ordovas M \& Schaefer EJ (1999) Estrogen increases apolipoprotein (apo) A-1 secretion in HepG2 cells by modulating transcription of the apoA-1 gene promoter. Arterioscler Thromb Vasc Biol 19, 2960-2965.

19. Lamon-Fava S (2000) Genistein activates apolipoprotein A-1 gene expression in the human hepatoma cell line HepG2. J Nutr 130, 2489-2492.

20. Sastry KN, Seedorf U \& Karthanasis SK (1988) Different cis-acting DNA elements control expression of the human apolipoprotein AI gene in different cell types. Mol Cell Biol 8, 605-614.

21. Harnish DC, Malik S, Kilbourne E, Costa R \& Karathanasis SK (1996) Control of apolipoprotein A-I gene expression through synergistic interaction between hepatocyte nuclear factors 3 and 4. J Biol Chem 271, 13621-13628.

22. Harnish DC, Malik S \& Karathanasis SK (1994) Activation of apolipoprotein A-I gene expression by the liver-enriched factor HNF-3. J Biol Chem 269, 28220-28226.

23. Kilbourne E, Widom R, Harnish DC, Malik S \& Karathanasis SK (1995) Involvement of early growth response factor Egr1 in apolipoprotein AI gene transcription. J Biol Chem 270, 7004-7010.

24. Ivanov GS, Kater JM, Jha SH, Stutius EA, Sabharwal R, Tricarico MD, Ginsburg GS \& Ozer JS (2003) Sp and GATA factors are critical for apolipoprotein AI downstream enhancer activity in human HepG2 cells. Gene 323, 31-42.

25. Zhang X, Jiao JJ, Bhavnani BR \& Tam SP (2001) Regulation of human apolipoprotein A-1 gene expression by equine estrogens. J Lipid Res 42, 1789-1800. 